\title{
Gut Vagal Afferents Differentially Modulate Innate Anxiety and Learned Fear
}

\author{
Melanie Klarer, ${ }^{1}$ Myrtha Arnold, ${ }^{1}$ Lydia Günther, ${ }^{2}$ Christine Winter, ${ }^{2}$ Wolfgang Langhans, ${ }^{1}$ and Urs Meyer ${ }^{1}$ \\ ${ }^{1}$ Physiology and Behavior Laboratory, ETH Zurich, 8603 Schwerzenbach, Switzerland and ${ }^{2}$ Department of Psychiatry and Psychotherapy, University \\ Hospital Carl Gustav Carus, Technical University Dresden, 01307 Dresden, Germany
}

\begin{abstract}
Vagal afferents are an important neuronal component of the gut-brain axis allowing bottom-up information flow from the viscera to the CNS. In addition to its role in ingestive behavior, vagal afferent signaling has been implicated modulating mood and affect, including distinct forms of anxiety and fear. Here, we used a rat model of subdiaphragmatic vagal deafferentation (SDA), the most complete and selective vagal deafferentation method existing to date, to study the consequences of complete disconnection of abdominal vagal afferents on innate anxiety, conditioned fear, and neurochemical parameters in the limbic system. We found that compared with Sham controls, SDA rats consistently displayed reduced innate anxiety-like behavior in three procedures commonly used in preclinical rodent models of anxiety, namely the elevated plus maze test, open field test, and food neophobia test. On the other hand, SDA rats exhibited increased expression of auditory-cued fear conditioning, which specifically emerged as attenuated extinction of conditioned fear during the tone re-exposure test. The behavioral manifestations in SDA rats were associated with region-dependent changes in noradrenaline and GABA levels in key areas of the limbic system, but not with functional alterations in the hypothalamus-pituitary-adrenal grand stress. Our study demonstrates that innate anxiety and learned fear are both subjected to visceral modulation through abdominal vagal afferents, possibly via changing limbic neurotransmitter systems. These data add further weight to theories emphasizing an important role of afferent visceral signals in the regulation of emotional behavior.
\end{abstract}

Key words: anxiety; fear; GABA; gut feelings; vagus nerve

\section{Introduction}

The CNS and viscera are engaged in constant bidirectional communication. This functional entity has been conceptualized as the gut-brain axis and allows top-down (CNS to viscera) and bottom-up (viscera to CNS) information flow (Mayer, 2011). One of the key neuronal elements of the gut-brain axis is the vagus nerve, whose afferent fibers are crucial for conveying visceral information to the brain (Berthoud and Neuhuber, 2000; Critchley and Harrison, 2013). Vagal afferent neurons synapse bilaterally on the nucleus tractus solitarius (NTS), from where visceral signals are transmitted to other brainstem nuclei and to various forebrain structures such as thalamus, hippocampus (HPC), amygdala (AMY), and prefrontal cortex (PFC; Berthoud and Neuhuber, 2000).

Vagal afferents may also be key neuronal elements mediating "gut feelings" (Forsythe et al., 2010; Mayer, 2011). Such feelings are typically conceptualized as somatic signals that influence de-

Received Jan. 20, 2014; revised March 17, 2014; accepted April 7, 2014.

Author contributions: M.K., W.L., and U.M. designed research; M.K., M.A., L.G., and C.W. performed research; M.K., M.A., L.G., C.W., W.L., and U.M. analyzed data; M.K., M.A., L.G., C.W., W.L., and U.M. wrote the paper.

This work was supported by The European Union's Seventh Framework Programme (FP7/2007-2011) under grant agreement 259679 awarded to U.M., and by funding granted by ETH Zurich, Switzerland (ETH Research Grant ETH-25_13-2) awarded to U.M. and W.L.

The authors declare no competing financial interests.

Correspondence should be addressed to Dr. Urs Meyer, Physiology and Behavior Laboratory, ETH Zurich, Schorenstrasse 19,8603 Schwerzenbach, Switzerland. E-mail: urmeyer@ethz.ch.

DOI:10.1523/JNEUROSCI.0252-14.2014

Copyright $\odot 2014$ the authors $\quad 0270-6474 / 14 / 347067-10 \$ 15.00 / 0$ cision making and behavioral responses without explicit awareness of the provoking cues (Damasio, 1996; Kahneman and Klein, 2009; Mayer, 2011). It is believed that these somatic signals originate primarily in the viscera and are transmitted to the CNS via endocrine and neuronal routes (Martin et al., 2004). Disruption of vagal signaling has generally been associated with a failure of the organism to convey gut-derived signals form the viscera to the CNS (Cryan and Dinan, 2012), and such deficits may also play a role in the precipitation of behavioral abnormalities involving altered mood and affect (Groves and Brown, 2005) and certain cognitive impairments (Clark et al., 1998, 1999). The clinical relevance of dysfunctional vagal activity is further reflected by the recent use of chronic vagus nerve stimulation (VNS) for treatment-resistant affective diseases (George et al., 2008; Wani et al., 2013).

Despite the existing evidence implicating vagal signaling in mood and affect, the relative involvement of vagal afferents versus efferents in the modulation of emotional functions often remains obscure. For example, current preclinical lesion models that aim to explore a role of the vagus nerve in emotional behaviors are virtually all based on total subdiaphragmatic vagotomy (TVX), in which both the afferents and efferents of the vagus nerve are surgically disconnected below the diaphragm (Bravo et al., 2011; Bercik et al., 2011). Not only does TVX induce severe side effects such as gastrointestinal secretory and motor dysfunctions, hypophagia, and subsequent body weight loss (Kraly et al., 1986), but it also does not allow for a discrimination of the relative functional contribution of vagal afferents versus efferents. 
A more selective and discriminative method to ascertain the functional properties of vagal afferents is surgical subdiaphragmatic vagal deafferentation (SDA; Norgren and Smith, 1994; Arnold et al., 2006). SDA is the most selective and complete method to disconnect vagal afferents as it eliminates all abdominal vagal afferents while sparing half of the vagal efferents (Norgren and Smith, 1994; Arnold et al., 2006). Here, we took advantage of the SDA model to explore for the first time whether preferential disconnection of vagal afferents is sufficient to modulate emotional behaviors and associated neurochemical parameters in rats.

\section{Materials and Methods}

Animals. Adult (280-320 g; presurgery body weight) male Sprague Dawley (Crl:CD) rats (obtained from Charles River) were used throughout the study. Upon arrival, the animals were assigned to group housing (two to four animals per cage) in acrylic, stainless-steel grid-floor cages (type IV, $595 \times 380 \times 200 \mathrm{~mm}$ ) and kept under a reversed light/dark cycle (lights on from 2000 to $0800 \mathrm{~h}$ ) at $22 \pm 2^{\circ} \mathrm{C}, 55-60 \%$ humidity. The animals had ad libitum access to water and standard rodent ground chow (Kliba 3436; Provimi Kliba Nafag) unless specified otherwise. Before surgery (see below), the animals were allowed to acclimatize to the new animal holding facility for 3 weeks, during which they were handled on a daily basis to habituate them to the experimenter. Daily animal handling was also continued after surgery until the commencement of behavioral testing (see below). All procedures were approved by the Cantonal Veterinarian's Office of Zurich.

SDA and Sham surgeries. Five days before surgery, rats were nursed with special diets, alternately consisting of unsweetened condensed milk (Migros) and wet mash (unsweetened condensed milk mixed with powdered ground chow, Kliba 3433; Provimi Kliba Nafag), to avoid excessive weight loss postsurgery (Arnold et al., 2006; Labouesse et al., 2012). This feeding regimen was continued until $5 \mathrm{~d}$ postsurgery, when the animals were re-adapted to standard rodent ground chow (Kliba 3436; Provimi Kliba Nafag) and given access to ad libitum chow thereafter.

The SDA surgery was adapted from the methods established by Norgren and Smith (1994). It consisted of a left-side intracranial vagal rhizotomy and a transection of the dorsal (left) subdiaphragmatic trunk of the vagus nerve according to procedures that have been frequently used and extensively validated in our laboratory (Porter et al., 1998; Arnold et al., 2006; Labouesse et al., 2012; Fig. 1). Sham surgery consisted of similarly exposing the vagal rootlets and dorsal subdiaphragmatic vagus, but without manipulating them. The SDA results in a complete disconnection of the abdominal afferents, while half of the abdominal vagal efferents are spared (Fig. 1). In previous attempts to induce complete vagal deafferentation through bilateral rhizotomy, we experienced that most animals with such lesions develop severe breathing complications after surgery, and as a consequence of this, they die within $12-48 \mathrm{~h}$ postsurgery (M. Arnold, unpublished observations). Given these limitations of bilateral rhizotomy, the SDA procedure is still the most complete and selective vagal deafferentation method existing to date.

Surgery was performed under sterile conditions and appropriate anesthesia. More specifically, the animals were pretreated subcutaneously with $50 \mu \mathrm{g} / \mathrm{kg}$ atropine (Sintetica, Mendrisio) 15-30 min before anesthesia, which consisted of intraperitoneal administration of a mixture of 5 $\mathrm{mg} / \mathrm{kg}$ xylazine (Rompun $2 \%$; Bayer AG) and $88 \mathrm{mg} / \mathrm{kg}$ ketamine (Ketamine Ratiopharm 50; Ratiopharm, Merckle). If required, additional ketamine was injected during surgery.

For the left-side intracranial vagal rhizotomy, the anesthetized animal was shaved from the chin caudally to the thorax and placed supine in an atraumatic head holder. A midline incision was then made from the anterior to the caudal mandible, and the skin was pulled away laterally with retractors. The left sternohyoid and omohyoid muscles were also retracted to expose the trachea and the external carotid artery. The occipital bone medial to the posterior lacerated foramen was then thinned with a dental drill and expanded with forceps without damaging the underlying dura. Subsequently, an incision was made in a mildly vascularized area above the ventral surface of the medulla, and the leaking CSF was absorbed. The dura was then retracted to expose the afferent and efferent vagal rootlets. The efferent rootlets were gently displaced to gain access to the afferent rootlets, the latter of which were then transected with forceps. Once the afferent nerve section was completed, the cavity was filled with sterile absorbable gelatin powder (Gelfoam; Pfizer,) to reduce further CSF leakage, and subsequently, the wound was closed in a single layer.

For the transection of the dorsal subdiaphragmatic trunk of the vagus nerve, the abdominal vagus nerve was exposed along the esophagus. For this purpose, a $3 \mathrm{~cm}$ midline laparotomy was made and the stomach was retracted using stay sutures that were placed through the corpus. Subsequently, the mesenteric connections between the ventral surface of the stomach and the liver were cut, and the right and central lobes of the liver were put aside rostrally and hold in place with gauze pads. The right (ventral) and left (dorsal) esophageal trunks were identified with the aid of a dissection microscope (Wild M650; Wild Heerbrugg), and the dorsal subdiaphragmatic trunk of the vagus nerve was then dissected via thermocautery. Approximately $5 \mathrm{ml}$ of warm Ringer's lactate solution (B. Braun) was injected into the abdomen during surgery. Following surgery, the midline laparotomy was closed using sutures, and $5 \mathrm{mg} / \mathrm{kg}$ carprofen (Rimadyl; E. Gräb AG) was injected subcutaneously for analgesia immediately after completion of the surgery and on each of the following $3 \mathrm{~d}$.

The body weight of each animal was monitored before surgery and for a period of 2 weeks postsurgery. The number of animals assigned to Sham and SDA surgery was $N=10$ and $N=12$, respectively.

Functional verification of SDA completeness. SDA completeness was verified using an established functional test ascertaining the lack of cholecystokinin (CCK) satiation that depends on intact abdominal vagal afferent fibers (Smith and Gibbs, 1985). The CCK test was conducted only after the animals had completed the behavioral and stress sensitivity tests (see below) because it involved implantation of intraperitoneal catheters and (temporary) single housing, both of which may undermine the reliability and robustness of the behavioral tests. Following appropriate recovery from catheter implantation, the animals were food deprived overnight $(13 \mathrm{~h}$ ) and injected with $4 \mu \mathrm{g} / \mathrm{kg}$ CCK-8 (Bachem) or vehicle (PBS) via the catheters using a within-subject crossover design. The injection of CCK or vehicle took place just before dark onset of the reversed $12 \mathrm{~h}$ dark/light cycle, after which the animals had ad libitum access to their food cups. Individual food intake was then monitored for $30 \mathrm{~min}$.

CCK- 8 treatment in Sham rats typically leads to a $25-40 \%$ reduction in food intake within the first $30 \mathrm{~min}$ after food presentation (Porter et al., 1998; Arnold et al., 2006; Labouesse et al., 2012). Therefore, the inclusion criterion for SDA rats was set at $25 \% \pm$ SEM CCK-induced reduction in food intake during the first $30 \mathrm{~min}$. Only data from animals that passed this functional verification were included in the final analysis and presentation of data. Using this criterion, only 1 of 12 SDA-lesioned animals had to be excluded from the final analysis, so that that the final number of animals was $N=10$ and $N=11$ for Sham and SDA, respectively.

Experimental test order and conditions. In keeping with the suggested role of vagal afferents in anxiety disorders (Groves and Brown, 2005; George et al., 2008), we investigated the effects of SDA on different forms of innate (unconditioned) anxiety-like behaviors and on learned fear. Behavioral testing commenced 2 weeks postsurgery and was conducted during the animals' active phase, that is, during the dark phase of the reversed light-dark cycle between 1000 and $1800 \mathrm{~h}$. The time of daily testing was counterbalanced across the two experimental groups. Throughout all behavioral testing, the animals were housed in groups of two to four animals per cage to avoid confounds arising from isolation stress (Zhang et al., 2012). All animals were tested repeatedly in a series of behavioral tests with an intertest recovery phase of 1-3 d. The order of behavioral tests was designed in such a way that the least stressful test was conducted at the beginning, and the most stressful test at the end of the test series. Hence, the following test order was used: (1) elevated plus maze test, (2) open field test, (3) food neophobia test, and (4) pavlovian fear-conditioning test (see below). The animals were fed ad libitum at the time of elevated plus maze and open field testing to minimize any interpretative limitations as to whether possible behavioral alterations in these tests might simply reflect changes in the desire to seek out and procure food. 
Upon completion of the behavioral analyses, we additionally conducted hormonal and neurochemical analyses to identify possible endocrine and neuronal mechanisms underlying the anticipated behavioral effects of SDA. Two weeks after the pavlovian fear-conditioning test, the animals were subjected to a stress sensitivity test (described below) to explore possible effects of SDA on the hypothalamus-pituitary-adrenal gland (HPA) stress response axis. One day after the stress sensitivity test, the animals were single caged and implanted with intraperitoneal catheters for the CCK satiation test (see above). Immediately after completion of the CCK test, the animals were recaged to the original groups of two to four animals per cage. Upon recaging to the original housing groups, animals were allowed a 2 week period for re-acclimatization to their peers and to fully recover from the preceding stress sensitivity test. Afterward, the animals were killed to collect fresh brain tissue for the subsequent postmortem neurochemical analyses (see below).

Elevated plus maze test. The elevated plus maze test is a widely used behavioral assay for measuring innate anxiety-like behavior in rodents (Belzung and Griebel, 2001; Cryan and Holmes, 2005). The test evaluates the animals' conflict between their natural tendencies to explore novel environments and to avoid unprotected open spaces.

The elevated plus maze was made of black acryl glass and elevated at a height of $85 \mathrm{~cm}$ above floor level. It consisted of four equally spaced arms (10 $\mathrm{cm}$ wide, $45 \mathrm{~cm}$ long) radiating out from a central square measuring $10 \times 10 \mathrm{~cm}$. Two opposing arms (enclosed arms) were enclosed by black walls $(40 \mathrm{~cm}$ high) from all sides except the side adjoining the central square. The other two arms (open arms) were exposed and the outer rim of the open arms was only guarded by a perimeter border of $1 \mathrm{~mm}$. Animals with high levels of innate anxiety are expected to spend less time in the unprotected open arms compared with animals with low levels of innate anxiety (Belzung and Griebel, 2001; Cryan and Holmes, 2005). The maze was located in a dimly lit experimental room. The light level in the open arms of the maze was balanced at 30 lux. A digital camera was mounted above the maze and images were transmitted at a rate of $5 \mathrm{~Hz}$ to a personal computer running the EthoVision (Noldus IT) software allowing the tracking of the animal's position.

To begin a trial, the animal was placed in the center of the maze facing one of the open arms. It was allowed to move freely for $5 \mathrm{~min}$ before being returned to the home cage. After each trial, the entire maze was cleansed with warm water. The levels of innate anxiety were indexed by the time spent and distance moved in the open arms, both of which were expressed as percentage scores over the total time spent and moved in all (open and enclosed) arms, respectively. An entry into an arm was defined by the spatial displacement of the animal's gravity center across the outlined zone borders and was automatically counted by the EthoVision tracking system. The total distance moved in the entire maze was also measured to analyze general locomotor activity during the test.

Open field test. The open field test is another widely used behavioral assay to evaluate innate anxiety-like behavior and locomotor responses to novel environments in rodents (Belzung and Griebel, 2001; Cryan and Holmes, 2005). The open field test was conducted in two identical square arenas $(80 \times 80 \mathrm{~cm})$ surrounded by walls $(50 \mathrm{~cm}$ high). The open field apparatus was made of gray Plexiglas and was located in a testing room under diffused lighting (30 lux as measured in the center of the arenas). A digital camera was mounted directly above the two arenas. Images were captured at a rate of $5 \mathrm{~Hz}$ and transmitted to a PC running the EthoVision (Noldus IT) tracking system.

The animals were gently placed in the one of the arena's corners and allowed to explore for $20 \mathrm{~min}$. After each trial, the apparatus was cleansed with warm water. For the purpose of data collection, the arena was conceptually partitioned into two zones: a center zone (measuring $25 \times 25$ $\mathrm{cm}$ ) in the middle of the arena and a peripheral zone occupying the remaining area. Animals with high levels of innate anxiety typically avoid the center zone and spend more time in close proximity to the walls (Belzung and Griebel, 2001; Cryan and Holmes, 2005). The dependent measures were the (1) number of entries into the center zone, (2) distance moved in the center zone, and (3) total distance moved in the entire arena. These dependent variables were measured during a 20 min test session and analyzed as a function of $5 \mathrm{~min}$ bins.
Food neophobia test. The food neophobia test of innate anxiety is based on the fact that rats, like most other rodents, typically avoid consuming novel foods (File, 2001). In this test, rats with high levels of innate anxiety typically take longer to begin eating an unfamiliar food compared with less anxious rats (File, 2001). Hence, latency to eat the novel food can be taken to index innate anxiety-like behavior.

The apparatus consisted of a rectangular arena $(78 \mathrm{~cm}$ long, $16 \mathrm{~cm}$ wide) surrounded by transparent walls ( $32 \mathrm{~cm}$ high). The apparatus was placed in a testing room under bright light (50 lux). A digital camera was mounted directly above the apparatus, and each test trial was recorded for subsequent video analyses by an experimenter who was blind to the experimental conditions.

The test was performed on 4 consecutive days: on the first $2 \mathrm{~d}$, the animals were placed into the test arena and allowed to freely explore it for 5 min once per day. This served to habituate the animals to the test apparatus and reduce potential confounds resulting from increased locomotor responses to novelty. The actual food neophobia test was then performed on the next 2 consecutive days, during which the novel food (sweetened Kellogg's Frosties cornflakes) was presented in the test arena. Each day included one test trial per animal, which began immediately after the animal was placed into the box and lasted for maximal $5 \mathrm{~min}$. If an animal started eating and continued for $3 \mathrm{~s}$, the trial was stopped and the animal was brought back into the colony room. If an animal did not start eating within the $5 \mathrm{~min}$ period, the trial was interrupted and the latency to eat was scored as $5 \mathrm{~min}$. On each day (including habituation days), subjects were food deprived for $16 \mathrm{~h}$ before placing them into the test apparatus.

After completion of a test trial, the animal was not brought back to its cage mates so as to avoid social transmission of food preferences (File, 2001). Instead, it was placed in a separate home cage (acrylic, stainlesssteel grid-floor cage, $595 \times 380 \times 200 \mathrm{~mm}$ ) where it received its standard food (rodent ground chow; Kliba 3436; Provimi Kliba Nafag) immediately after completion of the test trial and finally met its peers after they accomplished the test trials too.

Pavlovian fear conditioning. A classical (pavlovian) fear-conditioning test was used to assess the acquisition and expression of learned fear. In this test, the animals learn to associate an initially neutral stimulus (tobe-conditioned stimulus, CS) with a consequential aversive stimulus (unconditioned stimulus, US), and as a result, they develop a conditioned fear response in the form of freezing behavior (Johansen et al., 2011).

The apparatus comprised two sets of test chambers to provide two distinct contexts (context A and B). The first set of chambers (context A) included two operant chambers (Coulbourn Instruments; Habitest), each installed in a ventilated, sound-insulated chest $(72 \mathrm{~cm}$ wide $\times 45 \mathrm{~cm}$ long $\times 45 \mathrm{~cm}$ high). The chambers of context A measured $30 \times 25 \times 40$ $\mathrm{cm}$ (wide $\times$ long $\times$ high) and were fitted with a parallel grid shock floor (16 parallel bars, spaced $2 \mathrm{~cm}$ apart from each other; E10-18RF; Coulbourn Instruments), through which scrambled shocks at $1 \mathrm{~s}$ duration and $0.5 \mathrm{~mA}$ intensity (generated by a shock scrambler; Coulbourn Instruments; Model E13-14) could be delivered. Scrambled footshocks provided the US. Illumination inside the chambers of context A was provided by a house light $(2.8 \mathrm{~W})$ positioned on the panel wall. The second set of chambers (context B) comprised two rectangular enclosures ( $30 \mathrm{~cm}$ wide $\times 25 \mathrm{~cm}$ long $\times 40 \mathrm{~cm}$ high), which rested on brown Plexiglas floor instead of parallel grid floors. They were placed in ventilated, sound-insulated chests $(72 \times 45 \times 45 \mathrm{~cm})$ that were illuminated by an infrared light source instead of visible light. The chambers of context $B$ also contained a source of a distinct scent (verbena essential oil; Farfalla) to further differentiate context B from context A.

All chambers contained a Sonalert unit (Model H12-02M-2.9), which could deliver a $2.9 \mathrm{kHz}$ tone measuring $\sim 86 \mathrm{~dB}_{\mathrm{A}}$. This provided the CS. In addition, a miniature digital camera was mounted $30 \mathrm{~cm}$ directly above the center of the area of interest. The output of the camera was fed to a multiplexer (YSQ-430; Sony) before being transmitted to a PC. Animals were continuously videotaped for off-line scoring of freezing, which was used as an index of conditioned fear and defined as a lack of all movement, except for respiratory-related movements (Blanchard and Blanchard, 1969). For all three phases of the test (see below), freezing 
behavior was measured second by second by an experimenter who was blind to the experimental conditions.

The pavlovian fear-conditioning test was adapted from protocols previously established in-house (Bast et al., 2003; Schwendener et al., 2009) and consisted of three phases, each separated by $24 \mathrm{~h}$ :

On the first day, all animals were given five separate CS-US (toneshock) pairings, presented at 90 s intervals, in context A. The first CS-US trial was presented after an initial $3 \mathrm{~min}$ acclimatization period to context A. In each pairing, the $1 \mathrm{~s}$ shock US immediately followed the $30 \mathrm{~s}$ tone CS. On the day of conditioning, the amount of freezing during the five occasions of tone presentation provided a measure for the evaluation of the acquisition of conditioned fear.

On the second day, the animals were returned to context A. They were placed in the test chamber for a period of $6 \mathrm{~min}$, during which conditioned fear toward the context was evaluated. The expression of conditioned fear toward the context was indexed as percentage time freezing across the $6 \mathrm{~min}$ period and analyzed as a function of $30 \mathrm{~s}$ bins.

On the third day, the expression of conditioned fear toward the tone-CS was assessed in a novel context B. The tone stimulus was administered $3 \mathrm{~min}$ after the animals were placed into the test chamber. The tone remained on for a period of $6 \mathrm{~min}$, to parallel the test period of context freezing. The expression of conditioned fear toward the tone-CS was indexed as percentage time freezing across the $6 \mathrm{~min}$ period and also analyzed as a function of $30 \mathrm{~s}$ bins.

Stress sensitivity test. We measured basal and stress-induced plasma corticosterone (CORT) levels to investigate whether SDA may change the main hormonal output of the HPA stress response system. The stress sensitivity test was adapted from Macrì et al. (2008). Stress was induced by exposure to $20 \mathrm{~min}$ restraint stress, during which the animals were confined to a transparent Plexiglas tube ( $8 \mathrm{~cm}$ in diameter) of adjustable length (maximal $26 \mathrm{~cm}$ ). The restrainers were fixed to a table, which was placed in a brightly lit room. Tail vein blood samples were collected immediately before stress exposure and at 20, 40, 80, and 120 min after the onset of stress exposure. All animals were habituated to the tail blood sampling posture (not including tail incisions) and blood sampling room on 2 preceding days. The animals' bodies were softly wrapped in a cotton towel with their tails exposed for tail incisions. Tail vein blood (0.2-0.4 $\mathrm{ml}$ for each time point) was collected into EDTA-coated tubes (Microvette CB300 K2E; Sarstedt). The stress sensitivity test was staggered so that blood sampling was always performed between 1100 and $1600 \mathrm{~h}$ (dark cycle from 0800 to $2000 \mathrm{~h}$ ) to circumvent confounds arising from hormonal fluctuations at dark/light cycle onsets. Five to six identical restrainers were used at the same time, and rats were restraint cagewise to avoid possible stress transfer to nonrestraint animals. After each round of blood sampling, the animals were brought back to the colony room.

The collected blood was stored on ice and then centrifuged at 4000 rpm for $5 \mathrm{~min}$ at $4^{\circ} \mathrm{C}$ to obtain plasma samples. The plasma was aliquoted and stored at $-20^{\circ} \mathrm{C}$ until the CORT assay was performed. Plasma CORT levels were determined using commercially available quantitative competitive ELISA kits (AssayPro) according to the manufacturer's instructions. The samples were run in duplicates and were read by absorbance at a wavelength of $450 \mathrm{~nm}$. The detection limit of the CORT assay was 6.6 $\mathrm{ng} / \mathrm{ml}$.

Postmortem neurochemistry. Postmortem neurochemical analyses were performed to explore possible neuronal mechanisms underlying the anticipated behavioral effects of SDA. Following appropriate recovery from the CCK test (see above), animals were decapitated using a rat guillotine to obtain fresh brain tissue for the subsequent neurochemical analyses. The brains were extracted from the skull within $<2$ min, snap frozen in $-30^{\circ} \mathrm{C}$ cold isopentane, and stored at $-80^{\circ} \mathrm{C}$ until required. Frozen coronal sections ( $1 \mathrm{~mm}$ ) were prepared at the following bregma coordinates according to Paxinos and Watson (1998): anterior-posterior $(+3.2$ to +2.2$),(+1.7$ to +0.7$),(-1.8$ to -2.8$),(-2.8$ to -3.8$)$, and $(-5.1$ to -6.1$)$.

Based on the behavioral changes identified in SDA rats, we predicted neurochemical abnormalities in key areas of the limbic system (Bannerman et al., 2004; Sotres-Bayon et al., 2006; Shin and Liberzon, 2010; Maren et al., 2013). Therefore, tissue samples from both hemispheres were processed from the ventral PFC (vPFC; including prelimbic and

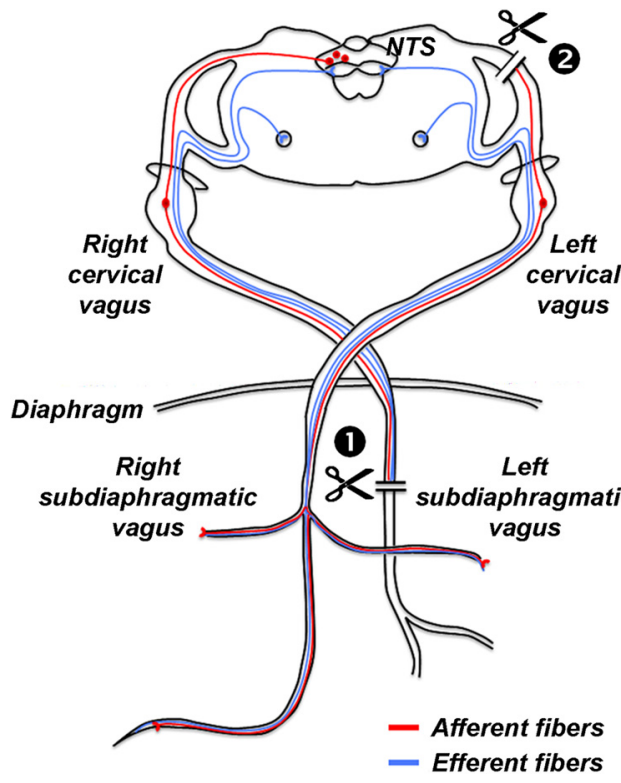

Figure 1. Schematic illustration of afferent and efferent vagal fibers targeted by the SDA procedure. Afferent and efferent fibers are represented in red and blue colors, respectively. In the SDA procedure, the left (dorsal) subdiaphragmatic trunk of the vagus nerve is fully transected (indicated by the first scissor symbol), leading to a disconnection of both afferent and efferent fibers in the left (dorsal) trunk of the vagus nerve. The right (ventral) subdiaphragmatic trunk of the vagus nerve is left intact. In addition, a left-sided intracranial vagal rhizotomy is performed (indicated by the second scissor symbol) to selectively disconnect the remaining vagal afferents. This SDA procedure induces a complete (100\%) disconnection of vagal afferents while leaving $50 \%$ of the vagal efferents functionally intact.

infralimbic cortices), nucleus accumbens (NAc; including shell and core subregions), AMY (including central and basolateral nuclei), and HPC (including dorsal and ventral CA1-CA3 regions) via micropunches of 1 $\mathrm{mm}$ diameter as previously described (Winter et al., 2009). In each of these brain areas, we measured the levels of noradrenalin (NA), serotonin (5-HT), and GABA. These neurotransmitters were selected based on their known involvement in regulating innate and conditioned forms of anxiety and fear (Sotres-Bayon et al., 2006; Shin and Liberzon, 2010; Johansen et al., 2011; Maren et al., 2013).

All neurotransmitter levels were measured using high performance liquid chromatography (HPLC). Tissue samples from each brain area of interest were homogenized by ultrasonication in $500 \mu \mathrm{l} 0.1 \mathrm{M}$ perchloric acid at $4^{\circ} \mathrm{C}$ immediately after processing. One hundred microliters of each homogenate was stored at $-80^{\circ} \mathrm{C}$ for subsequent protein determination. The remaining homogenates were centrifuged at $17,000 \times g$ and $4^{\circ} \mathrm{C}$ for $10 \mathrm{~min}$. Aliquots of each supernatant were added to equal volumes $(20 \mu \mathrm{l})$ of $0.5 \mathrm{M}$ borate buffer and stored at $-80^{\circ} \mathrm{C}$ for subsequent analyses of GABA. The remaining supernatants were used for immediate measurement of NA and 5-HT: perchloric acid extracts were separated on a column (Prontosil 120-C18-SH, $5 \mu \mathrm{m}, 200 \times 2 \mathrm{~mm}$; Bischoff Analysentechnik und -Geräte) at a flow rate of $0.3 \mathrm{ml} / \mathrm{min}$. The mobile phase consisted of $80 \mathrm{~mm}$ sodium dihydrogen phosphate, $0.9 \mathrm{~mm}$ octane-1sulfonic acid sodium salt, $0.5 \mathrm{~mm}$ EDTA disodium salt, $0.92 \mathrm{~mm}$ phosphoric acid, and 4\% 2-propanol (Merck). Monoamines were detected using an electrochemical detector (Decade II; Antec Leyden) at an electrode potential of $0.65 \mathrm{~V}$. For calibration, $0.1 \mathrm{~m}$ perchloric acid containing $10 \mathrm{~nm}$ NA and 5-HT were injected into the HPLC system before and after sample analysis. Sample analysis was performed based on peak areas using a computer-based chromatography data system (LCSolution Version 1.23; Shimadzu) in relation to the mean of the applied calibration solutions. GABA was precolumn derivatized with o-phthalaldehyde-2mercaptoethanol using a refrigerated autoinjector and then separated on a HPLC column (Prontosil C18 ace-EPS) at a flow rate of $0.6 \mathrm{ml} / \mathrm{min}$ and a column temperature of $40^{\circ} \mathrm{C}$. The mobile phase consisted of $50 \mathrm{~mm}$ sodium acetate, $\mathrm{pH} 5.7$, in a linear gradient from 5 to $21 \%$ acetonitrile. 
A

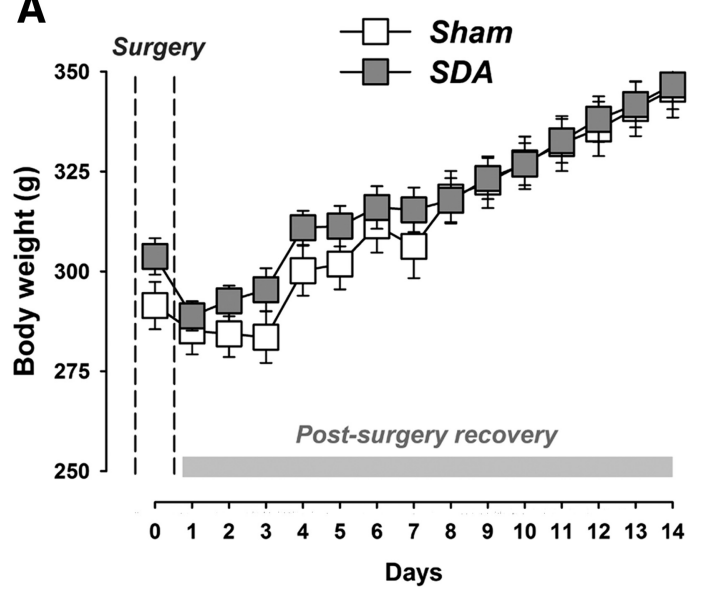

B

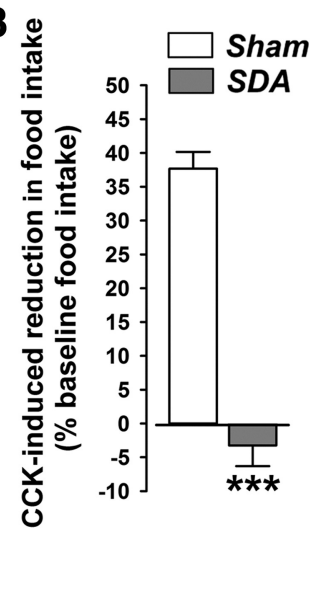

Figure 2. Body weights and functional verification of the SDA procedure. $\boldsymbol{A}$, The line plot shows body weights before surgery (day 0 ; indicated by the dashed lines) and during the postsurgery recovery period (days $1-14$ ). Note that both Sham and SDA rats similarly regained their initial presurgery body weight within $4 \mathrm{~d}$ after surgery. No significant group differences were detected between SDA and Sham rats. $N($ Sham $)=10, N(S D A)=12$; all values are means \pm SEM. $\boldsymbol{B}$, Verification of the SDA completeness in a CCK satiation test. The bar plot shows the percentage (\%) reduction in CCKinduced food intake (\% baseline food intake) during a 30 min test session. Note that SDA rats displayed no reduction in food intake in response to CCK administration relative to their baseline food intake, confirming the completeness of SDA. In contrast, Sham control rats displayed a $25-40 \%$ reduction in food intake in response to CCK relative to their baseline food intake. Against these backgrounds, 1 SDA animal (with 23\% reduction in food intake in response to CCK) of 12 was excluded from the final analysis and presentation of the data. ${ }^{* *} p<0.001 ; N($ Sham $)=10, N(\mathrm{SDA})=11$. All values are means \pm SEM.
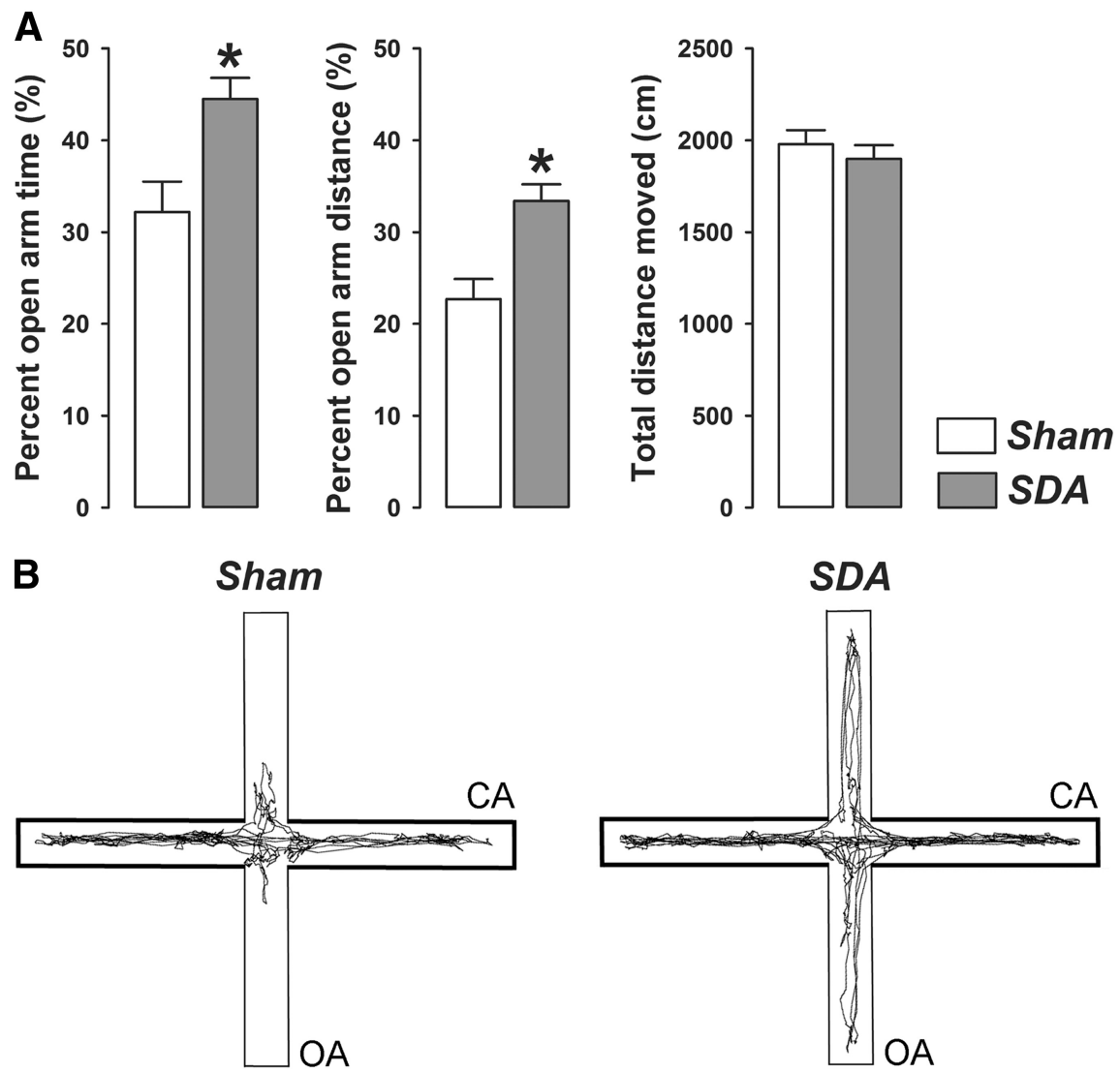

Figure 3. SDA reduces innate anxiety-like behavior in the elevated plus maze test. $A$, The bars show the percentage open arm time, percentage open arm distance, and total distance moved $(\mathrm{cm})$ in SDA and Sham control rats. $\boldsymbol{B}$, Computer-generated exploration paths of representative Sham and SDA rats in the elevated plus maze test. $C A$, enclosed (protected) arms; $0 A$, open (unprotected) arms. $N($ Sham $)=10, N(S D A)=11 ;{ }^{*} p<0.05$. All values are means \pm SEM.

Derivatized GABA was detected by its fluorescence at $450 \mathrm{~nm}$ after excitation at $330 \mathrm{~nm}$ (Winter et al., 2009).

Statistical analyses. Body weights were analyzed using a $2 \times 15$ (lesion $\times$ days) repeatedmeasures (RM) parametric ANOVA. The CCK-induced reduction in food intake was expressed as percentage basal food intake and analyzed using Student's $t$ test (two tailed). All data obtained in the elevated plus maze test were analyzed using Student's $t$ tests (two tailed). The dependent measures in the open field test and food neophobia test were analyzed by $2 \times 4$ (lesion $\times 5$ min bins) and $2 \times 2$ (lesion $\times$ trials) RM-ANOVA, respectively. The data obtained in the food neophobia test were first subjected to square root (sqrt) transformation to better conform to the assumption of data homogeneity and normality by parametric ANOVA. The dependent measures obtained during the acquisition phase of the pavlovian fear-conditioning test were analyzed using a $2 \times 5$ (lesion $\times$ trials) RM-ANOVA, and the data obtained in the context and tone test phases of the pavlovian fear-conditioning test were analyzed using $2 \times 12$ (lesion $\times 30 \mathrm{~s}$ bins) RM-ANOVAs. Plasma CORT levels in the stress sensitivity test were analyzed using $2 \times 5$ (lesion $\times$ sampling interval) RM-ANOVAs. All ANOVAs were followed by Fisher's least significant difference post hoc comparisons or restricted ANOVAs whenever appropriate. The neurochemical data were first analyzed using MANOVA, followed by Fisher's post hoc tests if appropriate. Statistical significance was set at $p<0.05$. All statistical analyses were performed using the statistical software IBM SPSS Statistics (version 19).

\section{Results}

Body weights and functional verification of SDA

Figure $2 A$ shows body weights before SDA or Sham surgeries and during the postsurgery recovery period. Sham and SDA rats similarly regained their initial presurgery body weight within $4 \mathrm{~d}$ after surgery, and no significant group differences in body weight were detected at any presurgical or postsurgical time point (Fig. 2A).

As expected (Porter et al., 1998; Arnold et al., 2006; Labouesse et al., 2012), Sham control rats displayed a $25-40 \%$ reduction in food intake in response to CCK relative to their baseline food intake (Fig. $2 B)$. In contrast, SDA rats displayed no such reduction in food intake in response to CCK administration relative to their baseline food intake, confirming the completeness of SDA (Fig. 2B).

\section{SDA reduces behavioral measures of innate anxiety}

In a first series of behavioral investigations, we assessed the effects of SDA on measures of innate anxiety. In the elevated 
plus maze test, SDA rats displayed a significant increase in the percentage time spent $(p<0.05)$ and percentage distance moved $(p<0.05)$ in the open arms compared with Sham rats (Fig. 3). SDA did not affect general locomotor activity as indexed by the total distance moved (Fig. 3). Hence, SDA rats exhibited a genuine reduction in anxiety-like behavior in the absence of confounding effects related to possible changes in basal locomotor activity.

Consistent with these results, SDA also caused a reduction in behavioral indices of innate anxiety in the open field test. As shown in Figure 4A, SDA rats displayed a significant increase in the center zone distance compared with Sham controls (main effect of lesion: $F_{(1,19)}=4.37, p<$ 0.05). The enhancement of center zone activity was particularly pronounced during the first $5 \mathrm{~min}$ of the open field test, as indicated by the significant interaction between lesion and bins $\left(F_{(3,57)}=3.29\right.$, $p<0.05)$ and the subsequent post hoc comparison confirming a significant $(p<$ 0.05) difference between SDA and Sham animals during the first $5 \mathrm{~min}$ bin (Fig. $4 A, D)$. Similarly, SDA rats entered the center zone more frequently than Sham rats especially during the first $5 \mathrm{~min}$ of the test, as indicated by the significant interaction between lesion and bins $\left(F_{(3,57)}=4.25\right.$, $p<0.01)$ and the subsequent post hoc comparison confirming a significant $(p<0.05)$ difference between SDA and Sham animals during the first $5 \mathrm{~min}$ bin (Fig. $4 B, D$ ). SDA did not affect the total distance moved in the entire open field arena (Fig. $4 C, D$ ). The total distance moved similarly decreased as a function of bins in SDA and Sham rats (main effect of bins: $F_{(3,57)}=180.44, p<$ 0.001 ), indicating normal locomotor habituation processes in both groups.

The food neophobia test provided additional evidence that SDA led to reduced behavioral indices of innate anxiety. In this test, the latency to eat a novel food was largely comparable in the first trial, but was markedly reduced in SDA rats compared with Sham rats in the second trial (Fig. 5). This trial-dependent effect of SDA indicates that the anxiolytic effect of SDA emerging in the second trial of the food neophobia test cannot simply be explained by changes in the drive to eat. The analysis of latency to eat led to a significant interaction between lesion and trials $\left(F_{(1,19)}\right.$ $=4.36, p<0.05)$. Subsequent analyses restricted to each group revealed a pronounced $(>60 \%)$ reduction in the latency to eat from trial 1 to 2 in SDA rats (main effect of trials: $F_{(1,10)}=24.66$, $p<0.001)$. Sham rats exhibited a much weaker $(\sim 25 \%)$ reduction from trial 1 to 2 (main effect of trials: $F_{(1,9)}=5.82, p<0.05$ ). Importantly, additional post hoc group comparisons confirmed that SDA rats displayed a significantly reduced latency to eat the novel food in the second trial compared with Sham rats $(p<$ 0.05, Fig. 5).

SDA attenuates extinction of auditory-cued conditioned fear To test whether vagal deafferentation may also modulate conditioned forms of anxiety/fear, we compared SDA and Sham rats in a classical (pavlovian) fear-conditioning test. The acquisition of
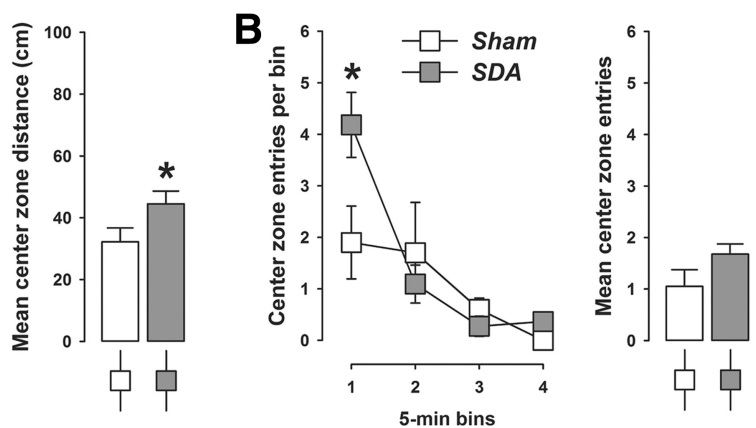

$\mathbf{D}$
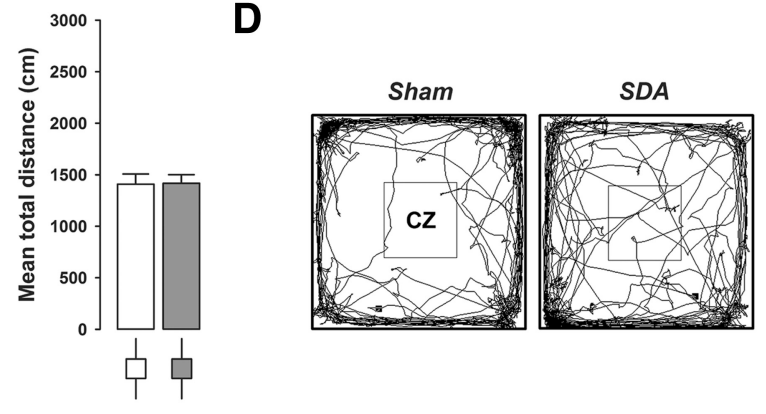

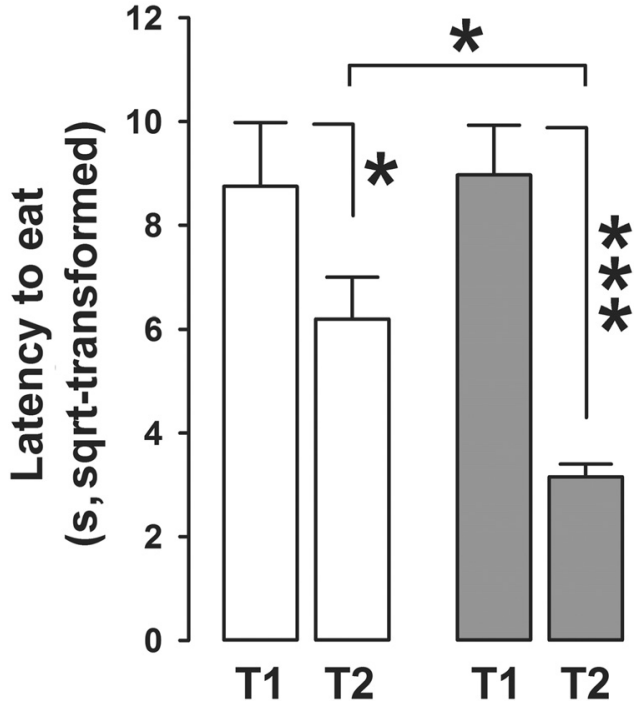

Figure 5. SDA reduces innate anxiety-like behavior in the food neophobia test. The graph shows latency (s, sqrt-transformed) to eat a novel food in trial (T) 1 and 2 of the food neophobia test for both SDA and Sham control rats. $N($ Sham $)=10, N($ SDA $)=11 ;{ }^{*} p<0.05$ and ${ }^{* * *} p<$ 0.001 . All values are means $\pm \mathrm{SEM}$.

conditioned fear during successive presentations of CS-US trials was highly comparable between SDA and Sham rats (Fig. 6A). Indeed, the amount of percentage time freezing during conditioning similarly increased in both groups as a function of CS-US trials (main effect of trials: $F_{(4,76)}=72.09, p<0.001$ ), indicating intact acquisition of the conditioned fear response to the auditory CS in both SDA and Sham animals. 

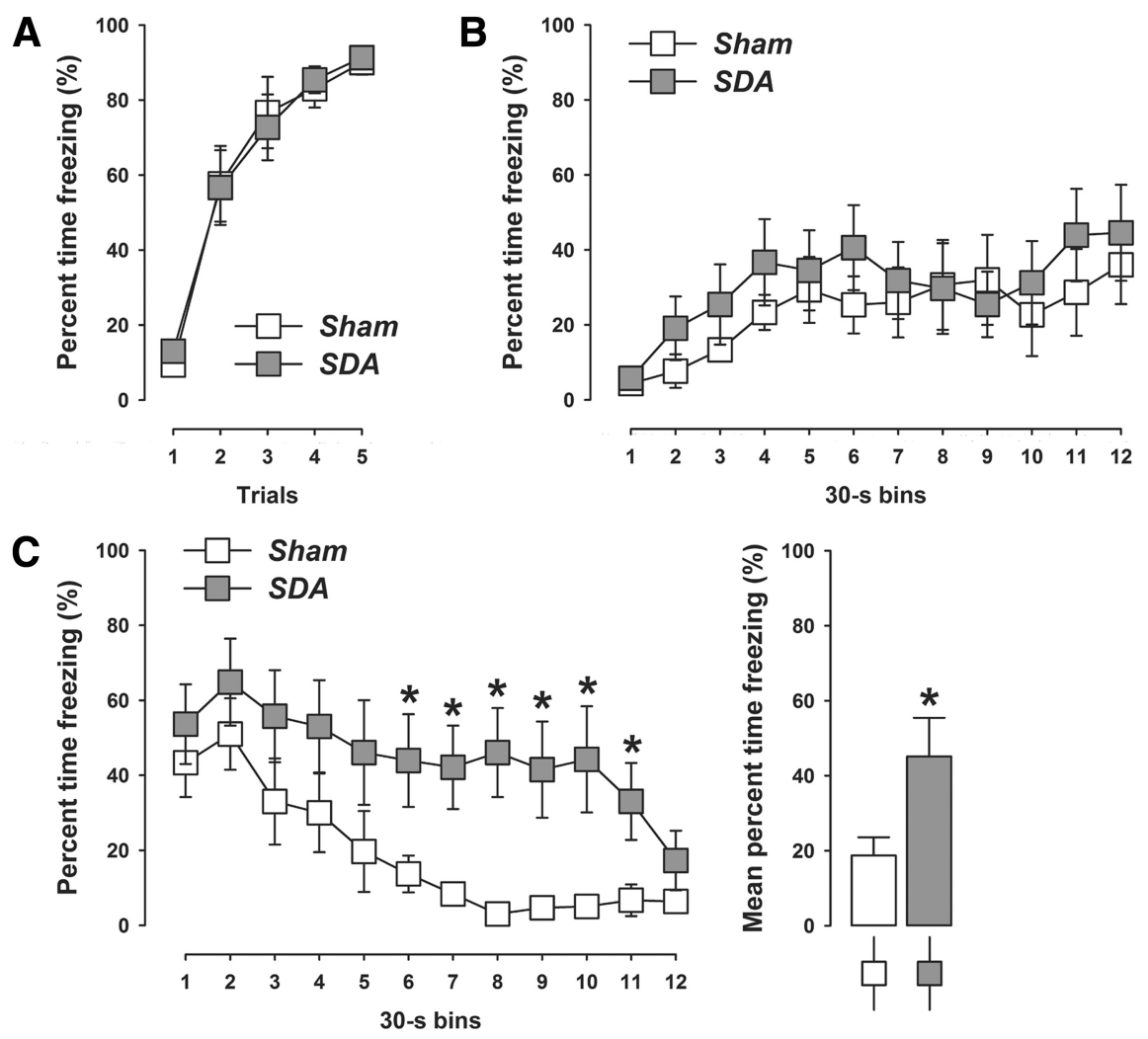

Figure 6. SDA attenuates extinction of auditory-cued conditioned fear. $A$, The line plot shows percentage time freezing during presentation of five successive CS-US trials during the conditioning phase of pavlovian fear conditioning in SDA and Sham control rats. $\boldsymbol{B}$, Expression of conditioned fear toward the context as measured by percentage time freezing during the context test. The line plot depicts percentage time freezing as a function of $30 \mathrm{~s}$ bins in SDA and Sham control rats. C, Expression of auditory-cued conditioned fear during the tone-CS test. The line plot shows percentage time freezing as a function of $30 \mathrm{~s}$ bins in SDA and Sham control rats. The bar plot depicts the mean percentage time freezing across all bins. $N($ Sham $)=10, N(\mathrm{SDA})=11 ;{ }^{*} p<0.05$. All values are means \pm SEM.

SDA did also not affect the expression of conditioned fear toward the context (Fig. 6B). In both SDA and Sham rats, percentage time freezing similarly increased as a function of bins during the 6 min context test period (see Fig. 6B). The analysis of context freezing only revealed a significant main effect of bins $\left(F_{(11,209)}=4.68, p<0.001\right)$.

Most interestingly, however, the expression of auditory-cued conditioned fear during the tone-CS test was significantly increased in SDA compared with Sham rats (Fig. $6 C$ ), as indicated by the significant main effect of lesion $\left(F_{(1,19)}=4.22, p<0.05\right)$. A closer inspection of the temporal development of auditory-cued conditioned fear revealed that the extinction rate of conditioned fear toward the tone-CS was attenuated in SDA compared with Sham rats. While the auditory-cued conditioned fear response was largely comparable shortly after the tone onset (i.e., within the first $60 \mathrm{~s}$ of CS presentation), the amount of percentage time freezing markedly subsided in Sham rats (Fig. 6C). Such extinction of auditory-cued conditioned fear was impaired in SDA rats, so that they persistently expressed high levels of freezing for a prolonged time (Fig. $6 C$ ). These results led to a significant interaction between lesion and bins $\left(F_{(11,209)}=2.10, p<0.05\right)$ in the analysis of percentage time freezing to the tone-CS. Post hoc group comparisons for individual bins confirmed that SDA rats displayed significantly increased auditory-cued conditioned fear in bins 6-11 compared with Sham rats (all p's $<0.05$; Fig. $6 C$ ).

\section{SDA modulates limbic neurotransmitter levels in the absence of altered CORT responses}

Following completion of the behavioral analyses, we conducted hormonal and neurochemical analyses to identify possible endocrine and neuronal factors that could underlie the SDA-induced modulation of innate anxiety and conditioned fear. Since alterations in HPA axis activity has often been associated with altered anxiety/fear responses (Franklin et al., 2012), we first evaluated whether SDA rats may exhibit altered basal and stressinduced CORT release. These investigations revealed no differences between SDA and Sham rats. Indeed, SDA and Sham rats displayed highly similar plasma levels of the stress hormone CORT, both under basal conditions and in response to restraint stress (Fig. 7). Exposure to restraint stress in SDA and Sham rats similarly increased plasma CORT levels, which peaked shortly after termination of stress exposure (i.e., between 20 and 40 min after stress onset) and decreased thereafter (Fig. 7). These time-dependent changes were reflected by the main effect of sampling interval $\left(F_{(4,76)}=70.38, p<\right.$ 0.001).

Next, we sought evidence for the possibility that the SDA-induced behavioral alterations may be paralleled by neurochemical changes in key areas of the limbic system. Our postmortem HPLC analyses indeed revealed that SDA led to brain region-specific changes in basal neurotransmitter levels (Fig. 8). Initial MANOVAs revealed significant effects for the vPFC (Wilks's $\Lambda=0.604, F_{(3,17)}=3.72$, $p<0.05$ ) and NAc (Wilks's $\Lambda=0.633, F_{(3,17)}=3.29, p<0.05$ ), but not for the AMY or HPC. In the vPFC region (Fig. 7A), SDA rats displayed significantly increased and decreased contents of GABA and NA, respectively, compared with Sham rats (both $p<$ $0.05)$. SDA animals also exhibited decreased levels of NA in the NAc relative to Sham controls $(p<0.05$; Fig. $8 B)$. The lesion did not significantly alter the levels of 5-HT in either the vPFC or in the NAc (Fig. $8 A, B$ ).

\section{Discussion}

Using the most complete and selective vagal deafferentation model existing to date, our study is the first to demonstrate a dual role of gut vagal afferents in the modulation of innate anxiety and learned fear. Rats with complete disconnection of abdominal vagal afferents consistently showed reduced innate anxiety-like behavior in three behavioral tests commonly used in preclinical rodent models of anxiety disorders (Belzung and Griebel, 2001; File, 2001; Cryan and Holmes, 2005). On the other hand, these animals displayed increased expression of auditory-cued fear conditioning, which was characterized primarily by an attenuation of conditioned fear extinction during the tone-CS test. These behavioral manifestations were accompanied by region-specific changes of basal neurotransmitter levels in key areas of the limbic system, suggesting that a disruption of the bottom-up (viscera to 
CNS) information flow by disconnection of the vagal afferents can exert imbalances in limbic neurochemistry.

Differential effects of SDA on innate anxiety and learned fear The differential effects of SDA on innate anxiety and conditioned fear might at first seem counterintuitive, if not contradictory. There are, however, a number of important distinctions between these behavioral domains, especially with respect to the underlying neuropsychological mechanisms. Innate (unconditioned) anxiety is typically defined as a state of goal conflict or uncertainty that does not entail any associative learning processes to allow its manifestation (McNaughton and Gray, 2000; Blanchard et al., 2001). On the other hand, the development of conditioned fear requires that the subject learns to associate an initially neutral stimulus (CS) with an aversive consequential event (McNaughton and Gray, 2000; Blanchard et al., 2001). Hence, the occurrence of the aversive and fear-inducing event is signaled and predicted by a CS. These distinctions have two important implications. First, unlike innate anxiety, conditioned fear is not driven by states of uncertainty or goal conflict, but rather by prediction and contingency (McNaughton and Corr, 2004). Second, the neuropsychological processes involved in conditioned fear and innate anxiety may even be viewed as diametrically opposite (McNaughton and Corr, 2004). Our data highlight that both of these neuropsychological domains are subjected to visceral modulation through vagal afferents.

At first glance, the effects of SDA on conditioned fear extinction may also seem incompatible with the lesion's effects in the food neophobia test. In the latter test, SDA rats displayed reduced latencies to eat the novel food specifically in the second trial, whereas SDA and Sham animals showed similar levels of neophobia during the first trial. This trial-specific effect in the food neophobia test may indicate that SDA facilitated extinction, whereas the extinction response displayed by SDA rats in the conditioned fear test strongly suggests the opposite. These seemingly paradoxical effects may be best explained when taking into account the aforementioned conceptual (and neuropsychological) differences between the tests of innate anxiety (in this case food neophobia) and learned fear (in this case Pavlovian fear conditioning): even though both tests appear to share some conceptual similarities in the sense that they involve an initial acquisition and subsequent extinction phase, they critically differ with respect to the extent to which a consequential stimulus (food or shock, US) is signaled by a discrete (but initially neutral, CS) stimulus. Against these backgrounds, our data indicate that SDA attenuates the extinction of conditioned fear responses, whereas it appears to facilitate the extinction of innate forms of anxiety.

Intriguingly, the attenuation of conditioned fear extinction by SDA directly complements recent seminal findings showing that VNS in rats increases the extinction rate of auditory-cued conditioned fear (Peña et al., 2013). Hence, activation and inhibition of vagal afferent values are means $\pm \mathrm{SEM}$.

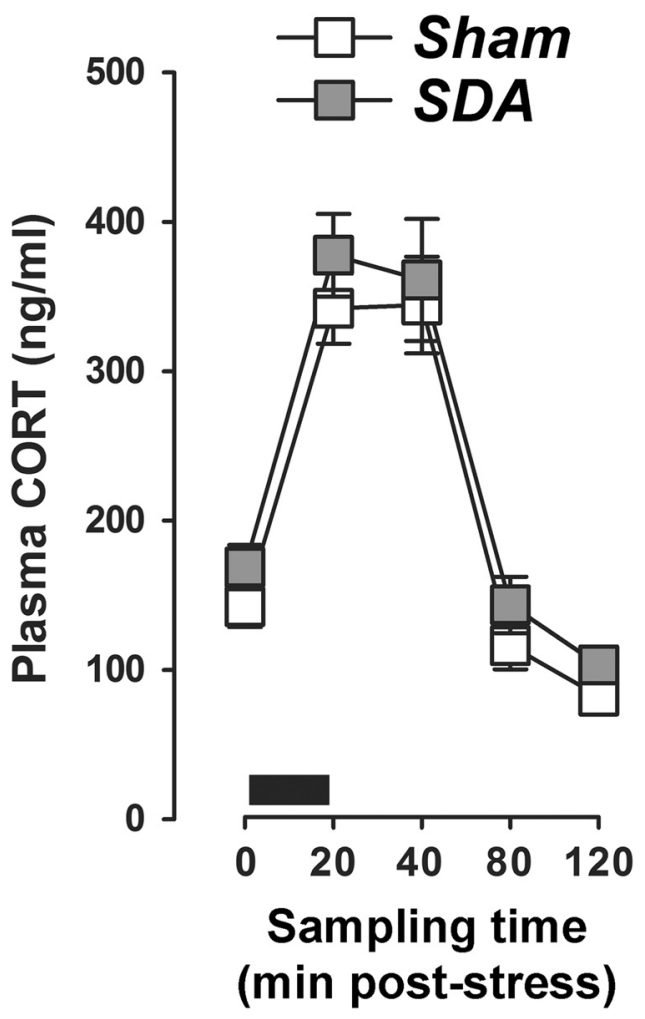

Figure 7. SDA does not affect basal and stress-induced CORT secretion. The graph shows plasma CORT levels at basal conditions (sampling time 0 ) as well as 20,40,80, and 120 min after onset of stress exposure. Stress was applied in the form of restraint stress for $20 \mathrm{~min}$ (indicated by the black bar). $N($ Sham $)=10, N(S D A)=11$. All values are means \pm SEM.
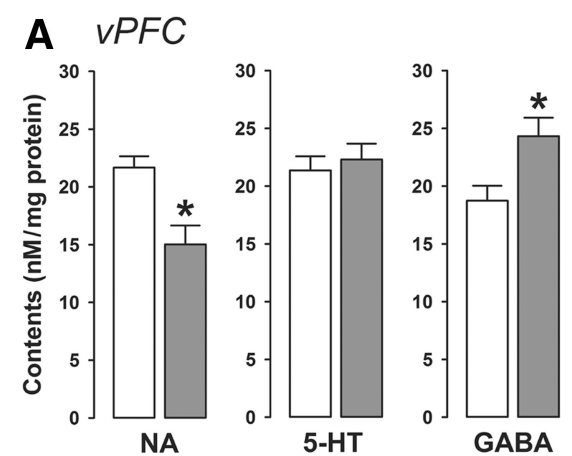

\section{C $A M Y$}
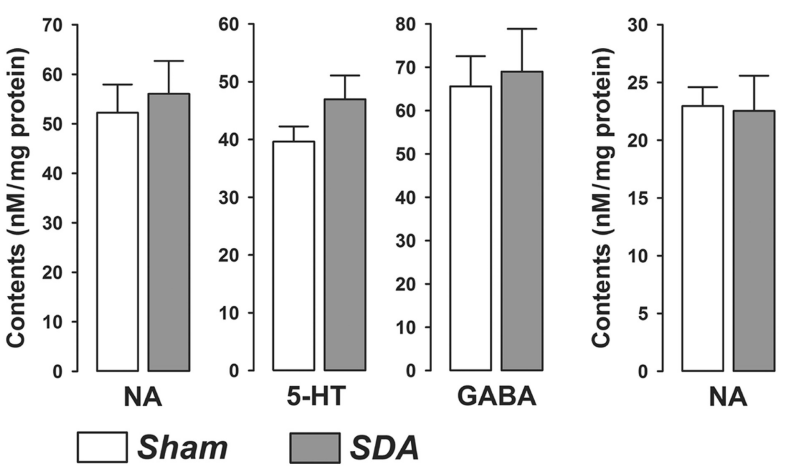

B NAC

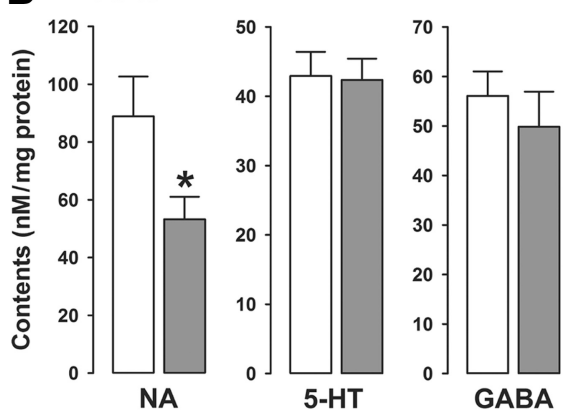

D $H P C$

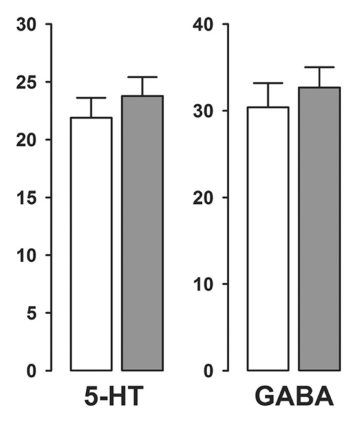

Figure 8. SDA modulates limbic neurotransmitter levels. Basal levels of NA, 5-HT, and GABA are depicted as nm/mg protein levels for the $(\boldsymbol{A}) \mathrm{vPFC},(\boldsymbol{B}) \mathrm{NAC},(\boldsymbol{C})$ AMY, and $(\boldsymbol{D})$ HPC of SDA and Sham control rats. $N($ Sham $)=10, N(S D A)=11 ;{ }^{*} p<0.05$. All 
signaling appears to facilitate and impair extinction of conditioned fear, respectively. Impaired extinction of learned fear is strongly associated with the development and/or persistence of stress-related disorders, especially post-traumatic stress disorder (PTSD) (Maren et al., 2013). Interestingly, individuals with PTSD have been reported to exhibit diminished vagal activity (Agorastos et al., 2013), but its functional consequences on fearrelated disturbances remain unknown. Together with the recent findings obtained in the VNS rat model (Peña et al., 2013), we deem our data encouraging for further studies exploring a role of vagal afferent signaling in PTSD and related disorders, including its potential therapeutic value for extinguishing acquired fear responses.

\section{Limbic neurotransmitter changes induced by SDA}

Within the CNS, limbic circuitries are believed to critically regulate both innate anxiety and conditioned fear (Bannerman et al., 2004; Sotres-Bayon et al., 2006; Johansen et al., 2011; Maren et al., 2013). Among the distinct limbic areas investigated, we found that SDA caused significant noradrenergic and GABAergic imbalances in the vPFC and NAc, while it spared these neurotransmitter systems in the AMY and HPC. Our neurochemical data thus suggest that the vPFC and NAc may be particularly sensitive to a disruption of visceral signaling induced by disconnection of the abdominal vagal afferents. Notably, the SDA-induced neurochemical imbalances are likely to be long-lasting because they were clearly noticeable at a time when all behavioral testing was completed. Furthermore, the neurotransmitter changes emerging in the limbic system of SDA rats likely represent secondary neurochemical responses to primary changes in up-stream neuronal signaling pathways that are directly targeted by vagal afferents. The primary target region of vagal afferents is the NTS, from where visceral signals are further conveyed to brainstem nuclei such as locus ceruleus and limbic structures such as HPC, AMY, and vPFC (Berthoud and Neuhuber, 2000). Additional research will be needed to delineate the neuronal routes by which SDA can lead to subsequent neurochemical changes in limbic forebrain structures such as the vPFC and NAc.

One clear limitation of our study is that we did not establish causal relationships between limbic neurochemical changes and the emergence of altered anxiety- and fear-related behaviors in the SDA model. Additional procedures, including in vivo microdialysis and pharmacological rescue approaches, are warranted in future investigations to explore causal links between the SDAinduced changes in emotional behavioral and limbic neurochemistry, especially in response to conflicting or stressful situations such as those present in tests of innate anxiety-like behavior or learned fear. On speculative grounds, however, a number of putative functional associations deserve attention. First, while the AMY and HPC play prominent roles in the acquisition and expression of auditory-cued and contextual fear responses, respectively (Phelps and LeDoux, 2005; Maren et al., 2013), the vPFC strongly regulates the extinction of conditioned fear (SotresBayon et al., 2006). With respect to the latter, there is a plethora of studies suggesting that inactivation of the PFC (especially its infralimbic portion) impairs extinction of conditioned fear (Morgan and LeDoux, 1995, 1999; Lebrón et al., 2004; Sotres-Bayon et al., 2006; Sierra-Mercado et al., 2011). Here, we found that SDA rats displayed a significant increase in prefrontal GABA levels, which in turn may have an inhibitory influence on vPFC activity, and therefore, may attenuate the extinction rate of auditory-cued fear (Sierra-Mercado et al., 2011; Morawska and Fendt, 2012). Indeed, our results match the findings of impaired conditioned fear extinction following intra-vPFC infusion of the $\mathrm{GABA}_{\mathrm{A}}$ receptor agonist muscimol (Sierra-Mercado et al., 2011; Morawska and Fendt, 2012).

In agreement with chronic VNS models in rats (Manta et al., 2013), we further revealed that SDA significantly decreased NA contents in the vPFC and NAc. NA is rapidly released in several limbic brain areas in response to acute stressors, and such augmentation of NA has been causally related to increased anxietylike behavior (Morilak et al., 2005). Accordingly, blockade of noradrenergic signaling exerts anxiolytic effects in rodent behavioral assays of innate anxiety (Morilak et al., 2005), similarly to what we observed in the present SDA model.

\section{Conclusions and outlook}

Our study shows that innate anxiety and learned fear are both subjected to visceral modulation through abdominal vagal afferents. These data add weight to theories emphasizing an important role of afferent visceral signals in the regulation of emotional behavior. Moreover, the emergence of attenuated fear extinction after SDA further highlights the importance of vagal afferent signaling for the remission of learned fear, which in turn may bear clinical relevance especially to the pathophysiology of PTSD and related disorders.

An emerging question that awaits exploration is whether the behavioral and neurochemical consequences of SDA may reflect a failure of the organism to convey gut-derived signals form the viscera to the CNS (Cryan and Dinan, 2012). This hypothesis is particularly intriguing in view of the recent findings demonstrating that germ-free mice lacking commensal gut-associated bacteria or mice receiving probiotic treatment with Lactobacillus rhamnosus (JB-1) show reduced innate anxiety-related behavior (Bravo et al., 2011; Diaz Heijtz et al., 2011). An alternative (but not mutually exclusive) mechanism may be that the SDAinduced effects on emotional behavior and neurochemistry may be linked to functional alterations in the sympathetic nervous system (King and Williams, 2009; Kerfoot and Williams, 2011). Future investigations of the possible links between SDA, gutderived signals, and/or sympathetic functions will readily help to gain more in-depth insights into the intricate mechanisms whereby visceral signals can influence complex brain and behavioral functions.

\section{References}

Agorastos A, Boel JA, Heppner PS, Hager T, Moeller-Bertram T, Haji U, Motazedi A, Yanagi MA, Baker DG, Stiedl O (2013) Diminished vagal activity and blunted diurnal variation of heart rate dynamics in posttraumatic stress disorder. Stress 16:300-310. CrossRef Medline

Arnold M, Mura A, Langhans W, Geary N (2006) Gut vagal afferents are not necessary for the eating-stimulatory effect of intraperitoneally injected ghrelin in the rat. J Neurosci 26:11052-11060. CrossRef Medline

Bannerman DM, Rawlins JN, McHugh SB, Deacon RM, Yee BK, Bast T, Zhang WN, Pothuizen HH, Feldon J (2004) Regional dissociations within the hippocampus-memory and anxiety. Neurosci Biobehav Rev 28:273-283. CrossRef Medline

Bast T, Zhang WN, Feldon J (2003) Dorsal hippocampus and classical fear conditioning to tone and context in rats: effects of local NMDA-receptor blockade and stimulation. Hippocampus 13:657-675. CrossRef Medline

Belzung C, Griebel G (2001) Measuring normal and pathological anxietylike behaviour in mice: a review. Behav Brain Res 125:141-149. CrossRef Medline

Bercik P, Park AJ, Sinclair D, Khoshdel A, Lu J, Huang X, Deng Y, Blennerhassett PA, Fahnestock M, Moine D, Berger B, Huizinga JD, Kunze W, McLean PG, Bergonzelli GE, Collins SM, Verdu EF (2011) The anxiolytic effect of Bifidobacterium longum NCC3001 involves vagal pathways for gut-brain communication. Neurogastroenterol Motil 23:1132-1139. CrossRef Medline 
Berthoud HR, Neuhuber WL (2000) Functional and chemical anatomy of the afferent vagal system. Auton Neurosci 85:1-17. CrossRef Medline

Blanchard DC, Griebel G, Blanchard RJ (2001) Mouse defensive behaviors: pharmacological and behavioral assays for anxiety and panic. Neurosci Biobehav Rev 25:205-218. CrossRef Medline

Blanchard RJ, Blanchard DC (1969) Crouching as an index of fear. J Comp Physiol Psychol 67:370-375. CrossRef Medline

Bravo JA, Forsythe P, Chew MV, Escaravage E, Savignac HM, Dinan TG, Bienenstock J, Cryan JF (2011) Ingestion of Lactobacillus strain regulates emotional behavior and central GABA receptor expression in a mouse via the vagus nerve. Proc Natl Acad Sci U S A 108:16050-16055. CrossRef Medline

Clark KB, Smith DC, Hassert DL, Browning RA, Naritoku DK, Jensen RA (1998) Posttraining electrical stimulation of vagal afferents with concomitant vagal efferent inactivation enhances memory storage processes in the rat. Neurobiol Learn Mem 70:364-373. CrossRef Medline

Clark KB, Naritoku DK, Smith DC, Browning RA, Jensen RA (1999) Enhanced recognition memory following vagus nerve stimulation in human subjects. Nat Neurosci 2:94-98. CrossRef Medline

Critchley HD, Harrison NA (2013) Visceral influences on brain and behavior. Neuron 77:624-638. CrossRef Medline

Cryan JF, Dinan TG (2012) Mind-altering microorganisms: the impact of the gut microbiota on brain and behaviour. Nat Rev Neurosci 13:701712. CrossRef Medline

Cryan JF, Holmes A (2005) The ascent of mouse: advances in modelling human depression and anxiety. Nat Rev Drug Discov 4:775-790. CrossRef Medline

Damasio AR (1996) The somatic marker hypothesis and the possible functions of the prefrontal cortex. Philos Trans R Soc Lond B Biol Sci 351: 1413-1420. CrossRef Medline

Diaz Heijtz R, Wang S, Anuar F, Qian Y, Björkholm B, Samuelsson A, Hibberd ML, Forssberg H, Pettersson S (2011) Normal gut microbiota modulates brain development and behavior. Proc Natl Acad Sci U S A 108:3047-3052. CrossRef Medline

File SE (2001) Factors controlling measures of anxiety and responses to novelty in the mouse. Behav Brain Res 125:151-157. CrossRef Medline

Forsythe P, Sudo N, Dinan T, Taylor VH, Bienenstock J (2010) Mood and gut feelings. Brain Behav Immun 24:9-16. CrossRef Medline

Franklin TB, Saab BJ, Mansuy IM (2012) Neural mechanisms of stress resilience and vulnerability. Neuron 75:747-761. CrossRef Medline

George MS, Ward HE Jr, Ninan PT, Pollack M, Nahas Z, Anderson B, Kose S, Howland RH, Goodman WK, Ballenger JC (2008) A pilot study of vagus nerve stimulation (VNS) for treatment-resistant anxiety disorders. Brain Stimul 1:112-121. CrossRef Medline

Groves DA, Brown VJ (2005) Vagal nerve stimulation: a review of its applications and potential mechanisms that mediate its clinical effects. Neurosci Biobehav Rev 29:493-500. CrossRef Medline

Johansen JP, Cain CK, Ostroff LE, LeDoux JE (2011) Molecular mechanisms of fear learning and memory. Cell 147:509-524. CrossRef Medline

Kahneman D, Klein G (2009) Conditions for intuitive expertise: a failure to disagree. Am Pshychol 64:515-526. CrossRef Medline

Kerfoot EC, Williams CL (2011) Interactions between brainstem noradrenergic neurons and the nucleus accumbens shell in modulating memory for emotionally arousing events. Learn Mem 18:405-413. CrossRef Medline

King SO 2nd, Williams CL (2009) Novelty-induced arousal enhances memory for cued classical fear conditioning: interactions between peripheral adrenergic and brainstem glutamatergic systems. Learn Mem 16:625634. CrossRef Medline

Kraly FS, Jerome C, Smith GP (1986) Specific postoperative syndromes after total and selective vagotomies in the rat. Appetite 7:1-17. CrossRef Medline

Labouesse MA, Stadlbauer U, Weber E, Arnold M, Langhans W, PachecoLópez G (2012) Vagal afferents mediate early satiation and prevent flavour avoidance learning in response to intraperitoneally infused exendin-4. J Neuroendocrinol 24:1505-1516. CrossRef Medline

Lebrón K, Milad MR, Quirk GJ (2004) Delayed recall of fear extinction in rats with lesions of ventral medial prefrontal cortex. Learn Mem 11:544548. CrossRef Medline

Macrì S, Chiarotti F, Würbel H (2008) Maternal separation and maternal care act independently on the development of HPA responses in male rats. Behav Brain Res 191:227-234. CrossRef Medline
Manta S, El Mansari M, Debonnel G, Blier P (2013) Electrophysiological and neurochemical effects of long-term vagus nerve stimulation on the rat monoaminergic systems. Int J Neuropsychopharmacol 16:459-470. CrossRef Medline

Maren S, Phan KL, Liberzon I (2013) The contextual brain: implications for fear conditioning, extinction and psychopathology. Nat Rev Neurosci 14:417-428. CrossRef Medline

Martin CO, Denburg NL, Tranel D, Granner MA, Bechara A (2004) The effects of vagus nerve stimulation on decision-making. Cortex 40:605612. CrossRef Medline

Mayer EA (2011) Gut feelings: the emerging biology of gut-brain communication. Nat Rev Neurosci 12:453-466. CrossRef Medline

McNaughton N, Corr PJ (2004) A two-dimensional neuropsychology of defense: fear/anxiety and defensive distance. Neurosci Biobehav Rev 28: 285-305. CrossRef Medline

McNaughton N, Gray JA (2000) Anxiolytic action on the behavioural inhibition system implies multiple types of arousal contribute to anxiety. J Affect Disord 61:161-176. CrossRef Medline

Morawska MM, Fendt M (2012) The effects of muscimol and AMN082 injections into the medial prefrontal cortex on the expression and extinction of conditioned fear in mice. J Exp Biol 215:1394-1398. CrossRef Medline

Morgan MA, LeDoux JE (1995) Differential contribution of dorsal and ventral medial prefrontal cortex to the acquisition and extinction of conditioned fear in rats. Behav Neurosci 109:681-688. CrossRef Medline

Morgan MA, LeDoux JE (1999) Contribution of ventrolateral prefrontal cortex to the acquisition and extinction of conditioned fear in rats. Neurobiol Learn Mem 72:244-251. CrossRef Medline

Morilak DA, Barrera G, Echevarria DJ, Garcia AS, Hernandez A, Ma S, Petre CO (2005) Role of brain norepinephrine in the behavioral response to stress. Prog Neuropsychopharmacol Biol Psychiatry 29:1214-1224. CrossRef Medline

Norgren R, Smith GP (1994) A method for selective section of vagal afferent or efferent axons in the rat. Am J Physiol 267:R1136-R1141. Medline

Paxinos G, Watson C (1998) The rat brain in stereotaxic coordinates. San Diego: Academic.

Peña DF, Engineer ND, McIntyre CK (2013) Rapid remission of conditioned fear expression with extinction training paired with vagus nerve stimulation. Biol Psychiatry 73:1071-1077. CrossRef Medline

Phelps EA, LeDoux JE (2005) Contributions of the amygdala to emotion processing: from animal models to human behavior. Neuron 48:175-187. CrossRef Medline

Porter MH, Hrupka BJ, Langhans W, Schwartz GJ (1998) Vagal and splanchnic afferents are not necessary for the anorexia produced by peripheral IL-1beta, LPS, and MDP. Am J Physiol 275:R384-R389. Medline

Schwendener S, Meyer U, Feldon J (2009) Deficient maternal care resulting from immunological stress during pregnancy is associated with a sexdependent enhancement of conditioned fear in the offspring. J Neurodev Disord 1:15-32. CrossRef Medline

Shin LM, Liberzon I (2010) The neurocircuitry of fear, stress, and anxiety disorders. Neuropsychopharmacology 35:169-191. CrossRef Medline

Sierra-Mercado D, Padilla-Coreano N, Quirk GJ (2011) Dissociable roles of prelimbic and infralimbic cortices, ventral hippocampus, and basolateral amygdala in the expression and extinction of conditioned fear. Neuropsychopharmacology 36:529-538. CrossRef Medline

Smith GP, Gibbs J (1985) The satiety effect of cholecystokinin. Recent progress and current problems. Ann N Y Acad Sci 448:417-423. CrossRef Medline

Sotres-Bayon F, Cain CK, LeDoux JE (2006) Brain mechanisms of fear extinction: historical perspectives on the contribution of prefrontal cortex. Biol Psychiatry 60:329-336. CrossRef Medline

Wani A, Trevino K, Marnell P, Husain MM (2013) Advances in brain stimulation for depression. Ann Clin Psychiatry 25:217-224. Medline

Winter C, Djodari-Irani A, Sohr R, Morgenstern R, Feldon J, Juckel G, Meyer U (2009) Prenatal immune activation leads to multiple changes in basal neurotransmitter levels in the adult brain: implications for brain disorders of neurodevelopmental origin such as schizophrenia. Int J Neuropsychopharmacol 12:513-524. CrossRef Medline

Zhang Y, Zu X, Luo W, Yang H, Luo G, Zhang M, Tang S (2012) Social isolation produces anxiety-like behaviors and changes PSD-95 levels in the forebrain. Neurosci Lett 514:27-30. CrossRef Medline 